Accepted at Cortex $16^{\text {th }}$ May 2022

\title{
Impaired sensitivity to spatial configurations in healthy aging
}

\author{
James Chard ${ }^{1}$, Richard Cook $^{1}$ and Clare Press ${ }^{1,2}$
}

1. Department of Psychological Sciences, Birkbeck, University of London

2. Wellcome Centre for Human Neuroimaging, UCL, UK

\section{Highlights}

- Older adults exhibit reduced sensitivity to configurations in faces and objects

- Configural difficulties exist alongside relatively preserved featural processing

- Modelling demonstrates reduced evidence accumulation, not altered response bounds

- Such perceptual decline can explain many social difficulties in healthy aging

- This pattern of difficulties has parallels in prosopagnosia and autism 


\section{Abstract}

Healthy aging is associated with decline in social, emotion, and identity perception, which is frequently attributed to deterioration of structures involved in social inference. It is believed that this decline is unlikely to be a result of perceptual aberrations due to intact (corrected) visual acuity. Nevertheless, the present studies examine whether more particular perceptual aberrations may be present in healthy aging, that could in principle contribute to such difficulties. The present study examined the possibility that particular deficits in configural processing impair the perception of faces in healthy aging. Across two signal detection experiments, we required a group of healthy older adults and matched younger adults to detect changes in images of faces that could differ either at the local, featural level, or in configuration of these features. In support of our hypothesis, older adults were particularly impaired in detecting configural changes, relative to detecting changes in features. The impairments were found for both upright and inverted faces and were similar in a task with images of inanimate objects (houses). Drift diffusion modelling suggested that this decline related to reduced evidence accumulation rather than a tendency to make configural judgments based on less evidence. These findings indicate that domain-general problems processing configural information contribute to the difficulties with face processing in healthy aging, and may in principle contribute to a range of higher-level social difficulties - with implications also for other groups exhibiting similar patterns in perception and understanding.

\section{Keywords}

Aging; face perception; visual perception; configural processing 


\section{Introduction}

A range of studies have indicated age-related difficulties in tasks involving face processing, including identity recognition (e.g., Boutet \& Faubert, 2006; Megreya \& Bindemann, 2015) and inferring affective and other internal states from facial expressions (e.g., Calder et al., 2003; Keightley et al., 2006; Kessels et al., 2014; see Ruffman et al., 2008 for a review). It has been proposed that such deficits produce a cascade of social difficulties, contributing to health and social problems in the aging population (Szanto et al., 2012). However, it is not known what underlies age-related deficits in face processing, and such understanding is a first step towards developing effective interventions.

Neurophysiological differences in the 'social brain' are frequently taken to explain particular difficulties with face perception and other social tasks in healthy aging (Ruffman et al., 2008; Fischer et al., 2010; Ziaei et al., 2019). The 'social brain' is a postulated network involving regions such as the orbitofrontal cortex, cingulate cortex and amygdala, and is implicated in the 'accurate perception of the dispositions and intentions of other individuals' (p. 28; Brothers, 1990). While changes in these circuits are likely to account for many social differences observed in healthy aging, there may be more domain-general perceptual loci that could alternatively or additionally explain difficulties with social tasks. Of course, if one finds it difficult to process perceptual cues (e.g., eye separation) necessary for deriving social information (e.g., that Henry is standing in front of me), social difficulties will necessarily ensue. Studies examining the nature of these difficulties frequently control for global perceptual acuity but these controls may be insufficient. 
Specifically, face perception difficulties cannot be primarily ascribed to simple acuity decline because they are frequently observed with relative preservation in other perceptual tasks, while there are also substantial individual differences in face perception amongst those with normal or corrected-to-normal vision (Wilmer, 2017). One possibility however is that older adults exhibit impaired processing of configurations - difficulties extracting 'second-order' information about the relationships between local features (Farah et al., 1998; Maurer et al., 2002). A deficit in this domain has been proposed to underlie face processing difficulties in other populations, such as in some individuals with developmental prosopagnosia (Barton et al., 2003; Avidan et al., 2011) and autism (Behrmann et al., 2006; Wallace et al., 2008), and therefore it represents a possible explanation of the perceptual difficulties seen in aging populations. While configural processing is of course required for a range of perceptual inferences, face perception is known to be especially reliant on it (Maurer et al., 2002). For instance, we can determine that the individual in front of us is Henry because of the particular eye separation, whereas we might most commonly recognise our friend's house due to the door colour.

There are reasons to hypothesise that older adults have relative deficits in processing configural compared with featural information, based on studies examining their perception of objects. For instance, the speed advantage observed in recognising global form in comparison with local features (the 'global precedence' effect) has been found to be reduced or even reversed in older adults (e.g., Lux et al., 2008; see also Wiese et al., 2013). At the neural level, the 'retrogenesis hypothesis' would also predict age-related alterations in circuitry involved in such processing. This hypothesis postulates that late-myelinated white matter is particularly vulnerable to decline (Brickman et al., 2013), which includes the inferior longitudinal fasciculus connecting occipital and temporal regions. Changes in the inferior 
longitudinal fasciculus have been noted in some individuals with developmental prosopagnosia (Thomas et al., 2009), who - as noted above - may have problems with configural processing, and it is thought to play a crucial role in a range of social and non-social perceptual processes (Benson et al., 1974).

To test whether older adults have a relative deficit in configural processing, Experiment 1 presented participants with pairs of face stimuli and required them to judge whether the configurations were the 'same' or 'different'. They also performed a control task where they made the same judgments about the features themselves. To test the domain-specificity of any impairments, we presented the same task with house stimuli, as well as inverted versions of all stimulus sets (note that recent studies have found featural processing to be disrupted by inversion, in the same way as configural processing; Murphy et al., 2020). We adopted a signal detection paradigm to allow us to isolate sensitivity from response bias. We hypothesised that, compared with younger adults, older adults' sensitivity to differences in configuration would be impaired relative to their sensitivity to featural differences. If this deficit was reflective of a domain-general problem with configural processing, it may be expected to apply across face and house stimuli, and across upright and inverted orientations (e.g., Susilo et al., 2013; Rossion, 2008). We also examined the precise nature of group differences with drift diffusion modelling of the data. Experiment 2 determined whether the effect in Experiment 1 replicated in an online setting and checked that the particular trial ordering was not responsible for effects.

\section{Experiment 1}

\section{Method}

\section{Participants}


Two groups participated, 30 younger adults aged 35 or under $\left(\mathrm{M}_{\text {age }}=23.50, \mathrm{SD}_{\text {age }}=4.27,20\right.$ females) and 30 older adults aged 60 or older ( $\mathrm{M}_{\text {age }}=71.07, \mathrm{SD}_{\text {age }}=6.32,23$ females $)$. Data on ethnicity and cultural background were not collected. The sample size was determined such that we would have at least $80 \%$ power to detect a medium-sized group $\mathrm{x}$ visual difference interaction effect $\left(\eta_{p}{ }^{2}=0.06, \alpha=0.05\right)$. Participants had normal or corrected-tonormal vision. The experiments were carried out in accordance with the ethical standards laid down in the 1964 Declaration of Helsinki and approved by the local Ethics Committee.

We obtained Weschler Abbreviated Scale of Intelligence (WASI) scores for two subtests (matrix reasoning and vocabulary) for all participants. Raw older adult scores ( $M=75.34 / 100$, with each test standardised to $/ 50 ; S D=8.45$ ) did not differ from raw younger adult scores $(\mathrm{M}=72.60 / 100, \mathrm{SD}=5.07), \mathrm{t}(58)=1.52, p=0.13$, indicating that any deficits observed in older adults in the main task are unlikely to have arisen from a decline in overall intellectual capabilities (note that raw scores provide a more appropriate comparison in the present context because FSIQ2 scores are normalised by age).

\section{Stimuli}

Stimuli were a set of nine images of faces and nine of houses, all based on the same two original images (stimulus set mirrored precisely that used by Yovel \& Kanwisher, 2004). From each base image, four additional versions were produced in which the features (eyes and mouth in the case of faces; windows and door in the case of houses) were replaced with those from other images while retaining the same second-order configuration. A further four additional images were produced where the features were unchanged from the original image, but second-order configuration was altered (increasing or reducing the horizontal distance between the eyes and vertical distance between the mouth and nose for faces; and 
the equivalent between windows and the door for houses; see Figure 1). All face stimuli had a neutral expression.

In Signal Absent trials, participants were presented with two identical images. In Signal Present trials, images differed either in respect of features or configuration. Given the number of images as described above, there were ten possible pairings of faces (and ten of houses) that differed in configuration, and a further ten that differed in features.

\section{Procedure}

Participants were seated in a dimly lit room at an approximate distance of $40 \mathrm{~cm}$ from a 24 inch LCD computer monitor (resolution $=1920 \times 1200$ pixels; refresh rate $=60 \mathrm{~Hz}$ ). Given the on-screen size of the stimuli and seating position, the visual angle was approximately $10^{\circ}$. The experiments were conducted in $\mathrm{MATLAB}^{\circledR}$ using the Cogent graphics toolbox. Before taking part, participants were informed of the basic procedure and were told that, where the images differed, they would do so only subtly. They were not, however, told anything about the nature of the differences that they may see. The task was calibrated via piloting by Yovel and Kanwisher (2004) to seek to achieve performance above chance levels whilst avoiding ceiling effects, and so involved relatively rapid presentation of images. In each trial, participants were shown a fixation dot in the centre of the screen $(500 \mathrm{~ms})$ followed by a blank screen (100 ms). The first image then appeared ( $250 \mathrm{~ms})$, followed by a fixation dot $(500 \mathrm{~ms})$, blank screen (100 $\mathrm{ms})$, and second image ( $250 \mathrm{~ms})$. Finally, they were asked whether the images were the 'same or different'. Participants responded using left and right keys, respectively. Participants received no feedback about whether individual responses were correct.

Participants completed 320 trials in total, consisting of 80 trials of each condition (upright face, inverted face, upright house and inverted house). Within each condition, 40 of the trials 
were Signal Present, of which half differed featurally and half configurally, and the remainder were Signal Absent. Half of the participants were presented with face blocks followed by house blocks, and the other half undertook the house blocks first. They undertook six trials before each new stimulus type to provide the opportunity to ask questions of the experimenter. These were randomly selected from the experimental set. Each block of 160 trials (houses or faces) was further divided into eight mini-blocks of 20 trials. Before each mini-block, participants were told whether images would appear upright or inverted. The start of each new block was controlled by the participant, enabling them to rest their eyes as required before continuing. Whether the first block was upright or inverted was counterbalanced between participants and thereafter the orientation of blocks alternated. Within the constraints described, presentation order was random. No feedback on participant performance was provided at any point in the experiment.

\section{Results}

$d^{\prime}$ 'was calculated as an index of sensitivity, reflecting the extent to which participants were more likely to report the presence of a probed stimulus when it was present than when it was absent, i.e., the difference between the z-scores of the hit rate (HR; proportion of Signal Present trials correctly identified) and false alarm rate (FAR; proportion of Signal Absent trials wrongly identified as Signal Present; $d^{\prime}=\theta^{-1}(H R)-\theta^{-1}(F A R)$; Kingdom \& Prins, 2010). A mixed ANOVA was conducted on the $d^{\prime}$ data (Fig. 1; all ps $>0.17$ in Levene's tests) with stimulus type (face or house), inversion (upright or inverted), and visual difference (featural or configural) as within-participants factors, and age group (younger adult or older adult) as a between-participants factor. 
We found some age-independent stimulus effects. Namely, there were significant main effects of stimulus type $\left(F(1,58)=39.69, p<0.001, \eta_{p}{ }^{2}=0.41\right)$ and inversion $(F(1,58)=120.30$, $\left.p<0.001, \eta_{p}{ }^{2}=0.68\right)$, qualified by an interaction between stimulus type and inversion $(F(1,58)$ $\left.=77.02, p<0.001, \eta_{p}^{2}=0.57\right)$. Specifically, while sensitivity for upright faces and houses did not differ $(t(59)=1.27, p=0.21)$, sensitivity towards inverted faces was lower than towards inverted houses $(t(59)=9.36, p<0.001)$.

There was also a significant main effect of age group $\left(F(1,58)=19.82, p<0.001, \eta_{p}{ }^{2}=0.26\right)$, and a borderline interaction between age group and inversion $\left(F(1,58)=4.00, p=0.05, \eta_{p}{ }^{2}=\right.$ $0.06)$, but no three-way interaction between stimulus, age group, and inversion $(F(1,58)=$ $0.02, p=0.89)$. Specifically, whilst older adult sensitivity was lower for both upright stimuli $(t(58)=2.81, p=0.01)$ and inverted stimuli $(t(58)=5.24, p<0.001)$, the impairment was relatively greater for inverted stimuli.

Most importantly for our hypotheses, there was an interaction between age group and visual difference $\left(F(1,58)=10.99, p<0.001, \eta_{p}^{2}=0.16\right)$. Whilst older adult sensitivity was lower for both featural differences $(t(58)=2.76, p=0.01)$ and configural differences $(t(58)=5.06, p<$ 0.001), older adults showed greater impairment in the configural task. There were no higher order interactions involving age group and visual difference (all $F s<2.13$, all $p s>0.15$ ). 

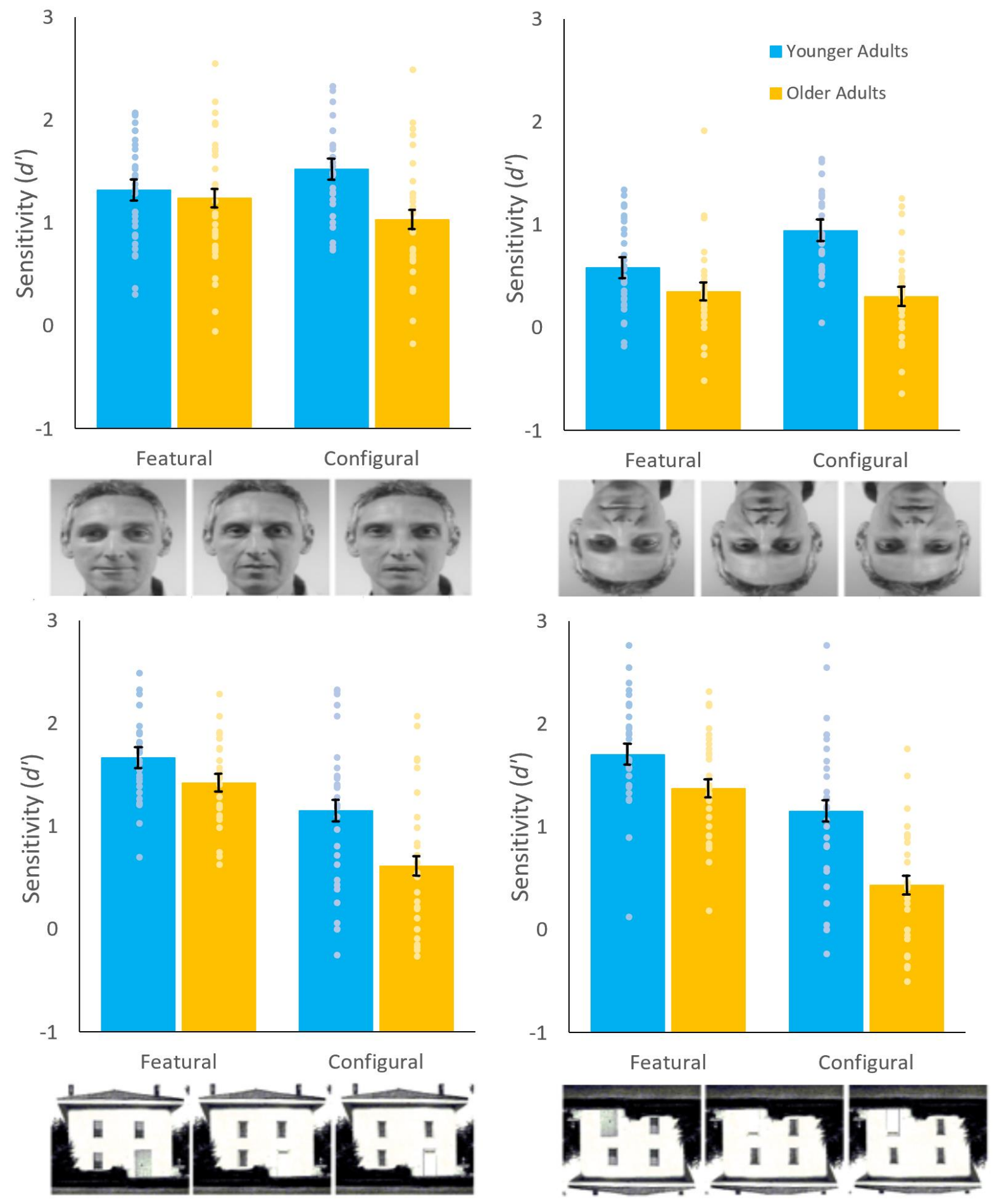

Figure 1: Sensitivity $\left(d^{\prime}\right)$ in Experiment 1 of younger adults and older adults to featural and configural differences in (clockwise from top left): (i) upright faces; (ii) inverted faces; (iii) inverted houses; (iv) upright houses. Example stimuli are shown below each chart, with the base image in the centre, featurally different image to the left and configurally different image to the right. The stimulus set was originally developed by Yovel \& Kanwisher, 2004 (the face stimuli are illustrative due to lack of written consent for the use of images from the original model in publications). 
In a signal detection paradigm, sensitivity differences could reflect a range of different perceptual processes that we aimed to distinguish with hierarchical drift diffusion modelling. Drift diffusion models (Ratcliff et al., 2016) treat decision making in a two-alternative forced choice procedure as involving sequential sampling of sensory evidence to compute a decision variable. When this accumulated decision variable meets a response boundary, the appropriate response is triggered.

We fitted a hierarchical drift diffusion model (hDDM) to responses using a package implemented in Python (Wiecki et al., 2013). This approach treats model parameters for each participant as being drawn from group level distributions, and uses Bayesian Markov Chain Monte Carlo (MCMC) sampling to estimate group and participant level parameters simultaneously. It parameterizes drift rate (v), representing efficiency of evidence accumulation; threshold (a), representing the extent of separation of decision making boundaries; and non-decision time $(\mathrm{t})$, representing processes not directly involved in stimulus discrimination, such as motor preparation to press the relevant response key. We allowed these parameters to vary based on age group and whether differences were featural or configural. We estimated models with 30,000 samples ('burn in' $=7500$ ), and models were compared using deviance information criteria as an approximation of Bayesian model evidence. Estimated parameters were then compared using a Bayesian significance test implemented in hDDM, which computes the posterior probability that group level parameters differ across conditions.

In both configural and featural trials, older adults exhibited lower drift rates, as well as greater boundary separation and non-decision time (all posterior probabilities for group differences > 0.99; higher values indicate a greater difference between conditions). Older adults exhibited 
a slower rate of accumulation of evidence relative to younger adults, but this was particularly marked for the configural task (mean drift rates: older adult configural $=0.402$; younger adult configural $=0.998$; older adult featural $=0.459$; younger adult featural $=0.636)$. This slower evidence accumulation resulted in lower d's despite more conservative decision thresholds, and longer non-decision times (noting that more conservative thresholds would tend to increase $d^{\prime}$ in this model). Furthermore, a partial correlation analysis showed a significant relationship between sensitivity and drift rate in the configural task, controlling for age and sensitivity and drift rate in the featural task $(r=0.596, N=60, p<0.001$; see Fig. 2 , and note that a correlation of the featural-configural difference between these parameters was similarly strong; $r=0.626, N=60, p<0.001$ ). In other words, the $d^{\prime}$ deficits reported in older adults reflect lower efficiency of extracting the perceptual evidence, rather than closer decision boundaries (e.g., greater prioritisation of speed than accuracy in the task - given that the boundaries were wider rather than closer). 

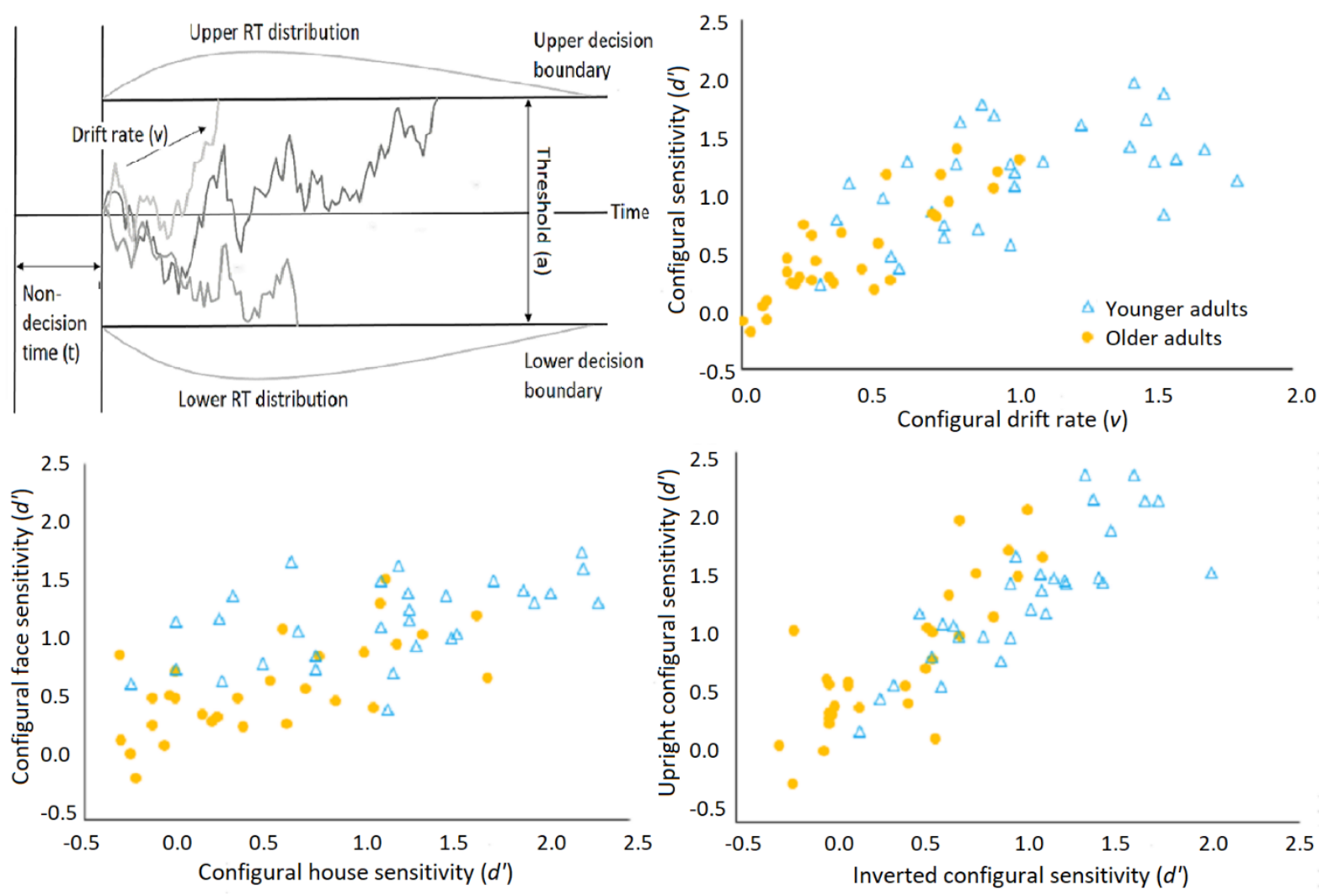

Figure 2: Experiment 1 modelling parameters (top) and individual differences between tasks (bottom). The top left panel illustrates the drift diffusion model parameters, whereby evidence is accumulated in the presence of Gaussian noise until a boundary is reached and response made. The positive drift rate $(v)$ towards the (correct) upper boundary represents the efficiency of evidence accumulation. The top right panel shows the correlation between sensitivity ( $d^{\prime}$ ) and drift rate ( $v$ ) towards configural differences in Experiment 1 . The bottom left panel shows the correlation between sensitivity towards configural differences in faces and houses. The bottom right panel shows the correlation between sensitivity towards configural differences in upright and inverted stimuli.

To examine further whether the age-related configural deficit was likely domain-general, we carried out correlation analyses to investigate how individual differences in each condition related to each other. Demonstrating that perceptual sensitivity towards configural differences in one condition related to that in others, there was a correlation between sensitivity towards configural differences in faces and in houses, when controlling for age and sensitivity towards featural differences in both stimuli types $(r=0.485, N=60, p<0.001)$. There was also a correlation between sensitivity to configural differences in upright and 
inverted stimuli, when controlling for age and sensitivity towards featural differences in both orientations $(r=0.785, N=60, p<0.001)$.

\section{Experiment 2}

Experiment 1 demonstrated lower sensitivity towards second-order configurations between features in older relative to younger adults, which was accounted for by reduced evidence accumulation in this group rather than narrower response boundaries. Experiment 2 was mainly designed to ensure the findings in Experiment 1 were robust. It also aimed to determine that a particular feature of the trial ordering in Experiment 1 was not responsible for the absence of face-specific deficits. Specifically, in Experiment 1, trials were blocked such that participants either completed all 160 trials with the house stimuli or all 160 with the face stimuli first. While the block type undertaken first was counterbalanced, it is possible that there is a domain-specific impairment to be found in older adults but that it is hard to detect in Experiment 1 due to differential fatigue or practice effects between groups. Experiment 2 therefore altered the procedure to involve short mini-blocks containing each stimulus type. It was carried out online due to the COVID pandemic.

\section{Method}

\section{Participants}

Two groups participated, 30 younger adults aged 35 or under $\left(\mathrm{M}_{\text {age }}=24.17, \mathrm{SD}_{\text {age }}=4.16,17\right.$ females) and 30 older adults aged 60 or older $\left(\mathrm{M}_{\text {age }}=68.00, \mathrm{SD}_{\text {age }}=4.76,21\right.$ females $)$. Participants were recruited using Prolific (www.prolific.co) and were selected on the basis of age group, having normal or corrected-to-normal vision, and having English as a first language. Data on ethnicity and cultural background were not collected. 


\section{Stimuli}

Stimuli were identical to those used in Experiment 1.

\section{Procedure}

The experiment was created and hosted using the Gorilla Experiment Builder (www.gorilla.sc, Anwyl-Irvine et al., 2020). Given the constraints of online recruitment, it was not possible to control the conditions in which participants undertook the experiment as closely as in Experiment 1, but participants were required to use a desktop or laptop computer and were instructed to complete the tasks in one sitting. Data on device used, screen resolution, and overall completion times were consistent with participants following these instructions. In relation to the reaction time data used for drift diffusion modelling, studies have indicated that online platforms including Gorilla provide a reliable measure (Anwyl-Irvine et al., 2021; Bridges et al., 2020).

The instructions and procedure for Experiment 2 reflected Experiment 1 except that practice trials were not included due to no experimenter being present to answer questions, and the 16 blocks of 20 trials were in a random order for each participant - with a message being shown before each block to inform participants whether the block would feature upright faces, inverted faces, upright houses, or inverted houses.

\section{Results}

We analysed Experiment 2 in the same way as Experiment 1, namely carrying out a mixed ANOVA with stimulus type, inversion, and visual difference as within-participants factors, and age group as a between-participants factor (see Fig.3; all ps $>0.20$ in Levene's tests). The first point of note is that the $d^{\prime}$ s are globally similar to those in Experiment $1(t(118)=0.31, p=$ 
0.76), suggesting that differences between the in-person and online contexts did not exhibit a major impact on participants' sensitivities.

Replicating Experiment 1, there were significant main effects of stimulus type $(F(1,58)=36.18$, $\left.p<0.001, \eta_{p}^{2}=0.38\right)$ and inversion $\left(F(1,58)=77.96, p<0.001, \eta_{p}^{2}=0.57\right)$, qualified by an interaction between stimulus type and inversion $\left(F(1,58)=31.79, p<0.001, \eta_{p}^{2}=0.35\right)$. Specifically, while sensitivity for upright faces and houses did not differ $(t(59)=0.66, p=0.51)$, sensitivity towards inverted faces was lower than towards inverted houses $(t(59)=9.08, p<$ $0.001)$.

There was also a significant main effect of age group $\left(F(1,58)=14.33, p<0.001, \eta_{p}^{2}=0.21\right)$. There was a trend towards an interaction between age group and inversion $(F(1,58)=3.35, p$ $=0.07)$, similar to that in Experiment 1 .

Most importantly for our hypotheses, and again replicating Experiment 1, there was an interaction between age group and visual difference $\left(F(1,58)=80.15, p<0.001, \eta_{p}{ }^{2}=0.58\right)$. Sensitivity towards featural differences did not differ between age groups $(t(58)=0.638, p=$ 0.64) but older adults were significantly less sensitive than younger adults to configural differences $(t(58)=6.68, p<0.001)$. It is noted that although the relative deficit was the same as in Experiment 1, Experiment 1 reflected older adults having a deficit in the featural conditions and a larger deficit in the configural condition whereas, in Experiment 2, OAs showed no featural deficit. Speculatively, this may be because older adults in the online study have more experience with computer-based tasks than many of their peers. Alternatively, it is possible that differences in viewing conditions in the online version (where the participants themselves determined elements like screen position and lighting) may have enabled older adults to improve their sensitivity, which removed the weaker featural deficit while leaving 

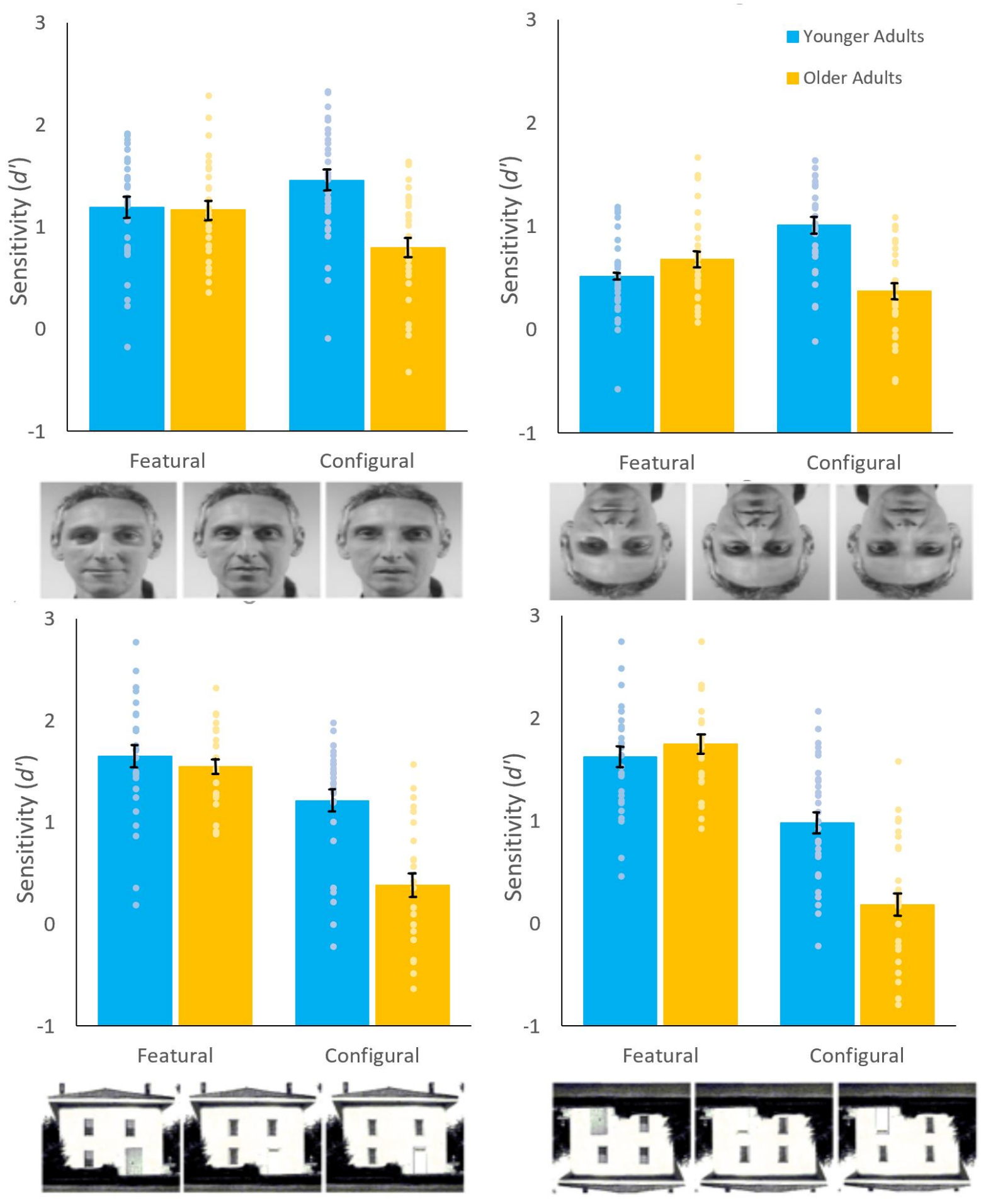

Figure 3: Sensitivity $\left(d^{\prime}\right)$ in Experiment 2 of younger and older adults to featural and configural differences in (clockwise from top left): (i) upright faces; (ii) inverted faces; (iii) inverted houses; (iv) upright houses. 
the interaction of interest intact. Regardless of the nature of this difference, the core questions relate to the relative difference between featural and configural processing between groups - which is the same in Experiments 1 and 2. There were no significant higher order interactions involving age group and visual difference (all Fs $<3.45$, all $p s>0.06$ ).
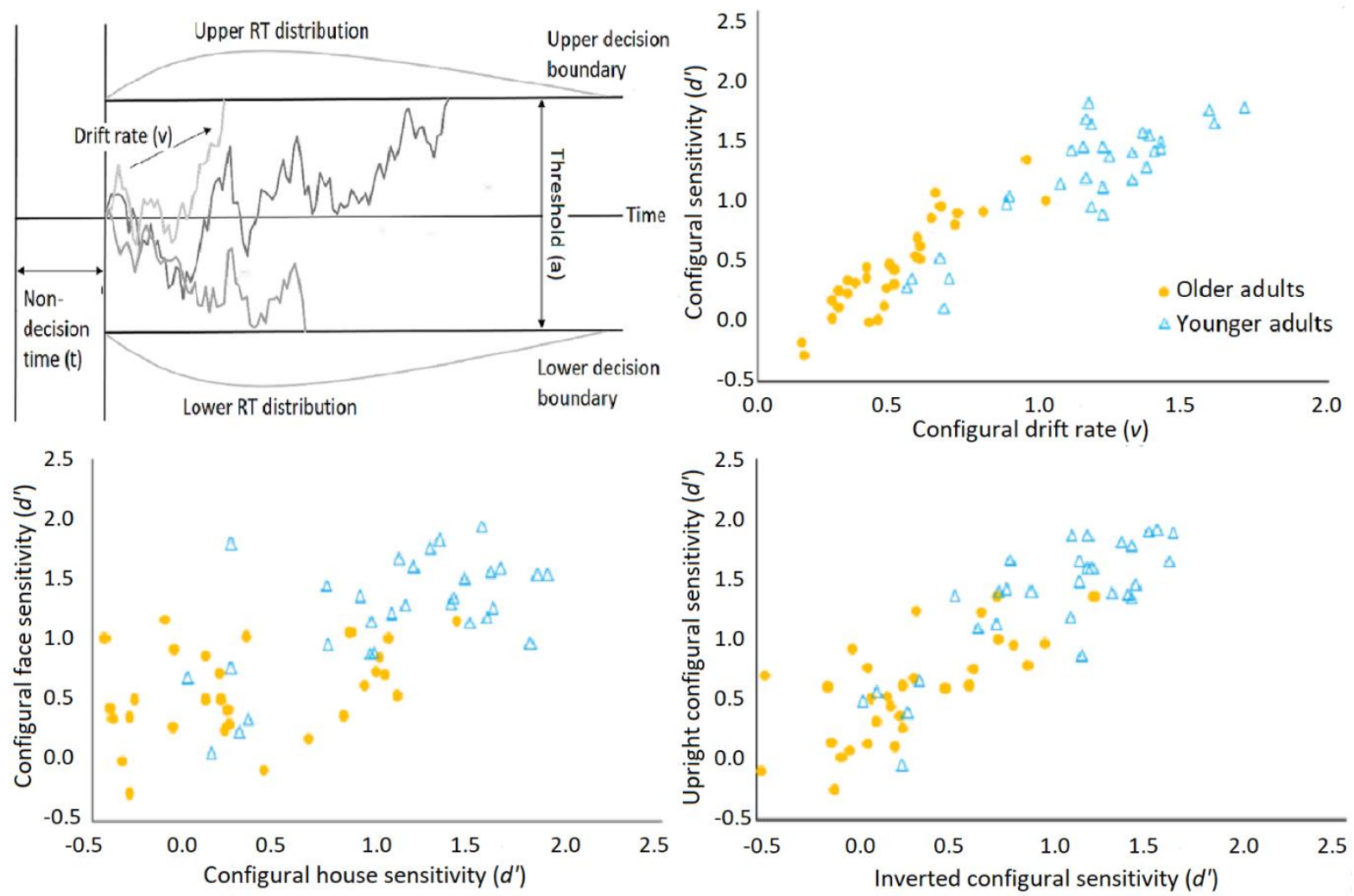

Figure 4: The top left panel illustrates the drift diffusion model parameters. The top right panel shows the correlation between sensitivity $\left(d^{\prime}\right)$ and drift rate $(v)$ towards configural differences in Experiment 2. The bottom left panel shows the correlation between sensitivity towards configural differences in faces and houses. The bottom right panel shows the correlation between sensitivity towards configural differences in upright and inverted stimuli.

We fitted a hDDM to responses using the same parameters as described in Experiment 1. As in Experiment 1, older adults' slower rate of evidence accumulation was particularly marked for the configural task (mean drift rates: older adult configural $=0.483$; younger adult configural $=1.151$; older adult featural $=1.047$; younger adult featural $=1.098$ ) resulting in lower $d^{\prime}$ 's despite more conservative decision thresholds and longer non-decision times. A 
partial correlation analysis showed a significant relationship between sensitivity and drift rate in the configural task, controlling for age and sensitivity and drift rate in the featural task ( $r=$ $0.841, N=60, p<0.001 ;$ see Fig. 4).

\section{Discussion}

The present studies demonstrate difficulties processing stimulus configurations in healthy aging. These difficulties were found across perception of faces and houses, and across upright and inverted orientations. They were also observed in the context of relatively intact processing of features of both faces and houses. Drift diffusion modelling suggested that deficits in configural sensitivity arose from less efficient evidence accumulation rather than from more liberal decision thresholds.

These results concur with observations that older adults exhibit difficulties determining the distance between facial features (e.g., Slessor et al., 2013) and determining horizontal compared with vertical spatial manipulations (Chaby et al., 2011). The present findings suggest that perceptual difficulties may emerge through impaired processing of the spatial configuration of features, rather than in generalised decline in visual processing that would be seen also when detecting featural aspects of images (see also Lux et al., 2008). The modelling further demonstrates that the atypicalities result from difficulties in evidence accumulation for configural features, rather than differential decision boundaries. The fact that the deficit was similar with houses suggests that the problem with processing configurations is not specific to faces (see also Chard et al., 2019), and also reduces the likelihood that specific features of the face stimuli determined effects - given that variation between faces and houses is greater than that within the category of faces. Observation of 
the deficit across upright and inverted images is consistent with evidence that faces can recruit qualitatively similar perceptual processing in both orientations (Murphy \& Cook, 2017; Susilo et al., 2013). This effect is also consistent with the idea that late-myelinated white matter is particularly vulnerable to age-related decline (Brickman et al., 2013), such that degeneration of the inferior longitudinal fasciculus connecting occipital and temporal regions may impact configural perception across domains. Further research into the pattern and rate of decline in configural processing across age, rather than simply comparing younger and older groups, could help in characterising the nature of the identified deficit.

The age-related impairment found in this study bears similarity to that proposed in autism (Behrmann et al., 2006; Wallace et al., 2008) and some cases of developmental prosopagnosia (Avidan et al., 2011; Gerlach et al., 2017) to explain face-processing difficulties (although see Joseph \& Tanaka, 2003; Thomas et al., 2009). Regardless of the nature of the relative deficits in the different populations, difficulties processing faces are accompanied by problems in relation to communication and social isolation in autism and developmental prosopagnosia, and studies have indicated analogous problems in older populations (Szanto et al., 2012). Such issues in older adults are, of course, likely related to a complex combination of situational factors tending to reduce social interaction (e.g. retirement, death of a partner, lower mobility; Vink et al., 2008), but the present study highlights an important contributor relating to cognitive decline. Traditionally, theories of identity recognition have emphasised the role of configural processing (Diamond \& Carey, 1986; Richler et al., 2011) and therefore difficulties with rapid and automatic recognition of individuals in healthy aging may relate to aberrant processing of configurations. Additionally, it is likely that configural processing is helpful in judging the emotional content of facial expressions, particularly those like anger and sadness where individual features are less informative in isolation (Smith et al., 2005; 
Bombari et al., 2013). Therefore, the difficulties processing configural information from faces may have a range of implications for the social understanding and interactions of older adults. By extension, such widespread social aberrations in other populations may be exacerbated, if not generated, by these quite particular perceptual difficulties.

There are some limitations to the present studies that should be noted. First, Experiment 2 was conducted online, which gives little control over viewing conditions. In principle, the lack of a featural deficit in older adults in Experiment 2 may be due to differences in viewing conditions between Experiments 1 and 2. Nevertheless, the broad pattern of results of interest for our hypotheses was replicated across in-person and online studies, supporting a growing body of evidence that online testing can be used effectively in cognitive science even for challenging psychophysical studies (Anwyl-Irvine et al., 2021; Bridges et al., 2020). Second, all stimuli were Caucasian and we did not collect ethnicity data pertaining to our participants. It is therefore important for future research to replicate these results with diverse face stimuli and diverse samples of younger and older adults. Importantly however, deficits in configural processing in the present studies were seen for objects (houses) and faces, and across orientations, and there was no reason to assume systematic differences in ethnicity between our older and younger adult groups, so it is unlikely that our findings are products of the so-called 'other race effect' (Sangrigoli et al., 2005).

In conclusion, older adults exhibit reduced sensitivity to visual configurations in faces and objects, reflecting reduced evidence accumulation for such information. Given evidence that configural processing plays a particular role in identity and emotion recognition from faces, and given the similarities with the patterns of deficits in autism and developmental 
prosopagnosia, the contribution of low-level visual deficits to social difficulties in healthy aging merits further examination. 


\section{Disclosure statement}

All dependent variables or measures that were analyzed for this article's target research question have been reported in the Methods section. All levels of all independent variables or all predictors or manipulations, whether successful or failed, have been reported in the Methods section. There were no excluded observations in the experiments in this article.

\section{Open practices statement}

This study was not formally preregistered. 


\section{References}

Anwyl-Irvine, A. L., Massonnié, J., Flitton, A., Kirkham, N., \& Evershed, J. K. (2020). Gorilla in our midst: An online behavioral experiment builder. Behavior Research Methods, 52(1), 388407.

Anwyl-Irvine, A., Dalmaijer, E. S., Hodges, N., \& Evershed, J. K. (2021). Realistic precision and accuracy of online experiment platforms, web browsers, and devices. Behavior Research Methods, 53(4), 1407-1425.

Avidan, G., Tanzer, M., \& Behrmann, M. (2011). Impaired holistic processing in congenital prosopagnosia. Neuropsychologia, 49(9), 2541-2552.

Barton, J. J., Cherkasova, M. V., Press, D. Z., Intriligator, J. M., \& O'Connor, M. (2003). Developmental prosopagnosia: A study of three patients. Brain and Cognition, 51(1), 12-30.

Behrmann, M., Thomas, C., \& Humphreys, K. (2006). Seeing it differently: visual processing in autism. Trends in Cognitive Sciences, 10(6), 258-264.

Benson, D. F., Segarra, J., \& Albert, M. L. (1974). Visual agnosia-prosopagnosia: A clinicopathologic correlation. Archives of Neurology, 30(4), 307-310.

Bombari, D., Schmid, P. C., Schmid Mast, M., Birri, S., Mast, F. W., \& Lobmaier, J. S. (2013). Emotion recognition: The role of featural and configural face information. The Quarterly Journal of Experimental Psychology, 66(12), 2426-2442.

Boutet, I., \& Faubert, J. (2006). Recognition of faces and complex objects in younger and older adults. Memory \& Cognition, 34(4), 854-864.

Brickman, A. M., Meier, I. B., Korgaonkar, M. S., Provenzano, F. A., Grieve, S. M., Siedlecki, K. L., ... \& Zimmerman, M. E. (2012). Testing the white matter retrogenesis hypothesis of cognitive aging. Neurobiology of Aging, 33(8), 1699-1715.

Bridges, D., Pitiot, A., MacAskill, M. R., \& Peirce, J. W. (2020). The timing mega-study: comparing a range of experiment generators, both lab-based and online. PeerJ, 8, e9414.

Brothers, L. (1990). The social brain: A project for integrating primate behaviour and neurophysiology in a new domain. Concepts in Neuroscience, 1, 27-51 
Calder, A. J., Keane, J., Manly, T., Sprengelmeyer, R., Scott, S., Nimmo-Smith, I., \& Young, A. W. (2003). Facial expression recognition across the adult life span. Neuropsychologia, 41(2), 195-202.

Chaby, L., Narme, P., \& George, N. (2011). Older adults' configural processing of faces: Role of second-order information. Psychology and Aging, 26, 71-79.

Chard, J., Edey, R., Yon, D., Murphy, J., Bird, G., \& Press, C. (2019). Atypical emotion recognition from bodies is associated with perceptual difficulties in healthy aging. Journal of Experimental Psychology: Human Perception and Performance, 45(6), 803-811.

Cook, R., \& Biotti, F. (2016). Developmental prosopagnosia. Current Biology, 26, R307-R318.

Diamond, R., \& Carey, S. (1986). Why faces are and are not special: an effect of expertise. Journal of Experimental Psychology: General, 115(2), 107-117.

Farah, M.J., Wilson, K.D., Drain, M., \& Tanaka, J.N. (1998). What is 'special' about face perception? Psychological Review, 105, 482-498.

Fischer, H., Nyberg, L., \& Bäckman, L. (2010). Age-related differences in brain regions supporting successful encoding of emotional faces. Cortex, 46, 490-497.

Gerlach, C., Klargaard, S.K., Peterson, A., \& Starrfelt, R. (2017). Delayed processing of global shape information in developmental prosopagnosia. PLoS One, 12, e0189253.

Keightley, M. L., Winocur, G., Burianova, H., Hongwanishkul, D., \& Grady, C. L. (2006). Age effects on social cognition: faces tell a different story. Psychology and Aging, 21(3), 558-572.

Kessels, R. P., Montagne, B., Hendriks, A. W., Perrett, D. I., \& de Haan, E. H. (2014). Assessment of perception of morphed facial expressions using the Emotion Recognition Task: Normative data from healthy participants aged 8-75. Journal of Neuropsychology, 8(1), 75-93.

Kingdom, F. A. A., \& Prins, N. (2010). Psychophysics: a practical introduction. London, UK: Elsevier.

Lux, S., Marshall, J. C., Thimm, M., \& Fink, G. R. (2008). Differential processing of hierarchical visual stimuli in young and older healthy adults: Implications for pathology. Cortex, 44(1), 2128. 
Maurer, D., Le Grand, R., \& Mondloch, C. J. (2002). The many faces of configural processing. Trends in Cognitive Sciences, 6(6), 255-260.

Megreya, A. M., \& Bindemann, M. (2015). Developmental improvement and age-related decline in unfamiliar face matching. Perception, 44(1), 5-22.

Murphy, J. \& Cook, R. (2017). Revealing the mechanisms of human face perception using dynamic apertures. Cognition, 169, 25-35.

Murphy, J., Gray, K. L., \& Cook, R. (2020). Inverted faces benefit from whole-face processing. Cognition, 194, 1-9.

Ratcliff, R., Smith, P. L., Brown, S. D., \& McKoon, G. (2016). Diffusion decision model: current issues and history. Trends in Cognitive Sciences, 20(4), 260-281.

Richler, J.J., Cheung, O.S., \& Gauthier, I. (2011). Holistic processing predicts face recognition. Psychological Science, 22, 464-471.

Rossion, B. (2008). Picture-plane inversion leads to qualitative changes of face perception. Acta Psychologica, 128(2), 274-289.

Ruffman, T., Henry, J. D., Livingstone, V., \& Phillips, L. H. (2008). A meta-analytic review of emotion recognition and aging: Implications for neuropsychological models of aging. Neuroscience \& Biobehavioral Reviews, 32(4), 863-881.

Sangrigoli, S., Pallier, C., Argenti, A.-M., Ventureyra, V.A.G., \& de Schonen, S. (2005). Reversibility of the other-race effect in face recognition during childhood. Psychological Science, 16, 440-444.

Slessor, G., Riby, D. M., \& Finnerty, A. N. (2012). Age-related differences in processing face configuration: The importance of the eye region. Journals of Gerontology Series B: Psychological Sciences and Social Sciences, 68(2), 228-231.

Smith, M. L., Cottrell, G. W., Gosselin, F., \& Schyns, P. G. (2005). Transmitting and decoding facial expressions. Psychological Science, 16(3), 184-189.

Susilo, T., Rezlescu, C., \& Duchaine, B. (2013). The composite effect for inverted faces is reliable at large sample sizes and requires the basic face configuration. Journal of Vision, 13, $1-9$. 
Szanto, K., Dombrovski, A. Y., Sahakian, B. J., Mulsant, B. H., Houck, P. R., Reynolds III, C. F., \& Clark, L. (2012). Social emotion recognition, social functioning, and attempted suicide in latelife depression. The American Journal of Geriatric Psychiatry, 20(3), 257-265.

Thomas, C., Avidan, G., Humphreys, K., Jung, K. J., Gao, F., \& Behrmann, M. (2009). Reduced structural connectivity in ventral visual cortex in congenital prosopagnosia. Nature Neuroscience, 12(1), 29-31.

Vink, D., Aartsen, M. J., \& Schoevers, R. A. (2008). Risk factors for anxiety and depression in the elderly: a review. Journal of Affective Disorders, 106(1-2), 29-44.

Wallace, S., Coleman, M., \& Bailey, A. (2008). An investigation of basic facial expression recognition in autism spectrum disorders. Cognition and Emotion, 22(7), 1353-1380.

Wiecki, T. V., Sofer, I., \& Frank, M. J. (2013). HDDM: hierarchical Bayesian estimation of the drift-diffusion model in Python. Frontiers in Neuroinformatics, 7, 14.

Wiese, H., Kachel, U., \& Schweinberger, S.R. (2013). Holistic face processing of own- and other-age faces in young and older adults: ERP evidence from the composite face task. Neuroimage, 74, 306-317.

Wilmer, J. B. (2017). Individual differences in face recognition: A decade of discovery. Current Directions in Psychological Science, 26(3), 225-230.

Yovel, G., \& Kanwisher, N. (2004). Face perception: domain specific, not process specific. Neuron, 44(5), 889-898.

Ziaei, M., Persson, J., Bonyadi, M. R., Reutens, D. C., \& Ebner, N. C. (2019). Amygdala functional network during recognition of own-age vs. other-age faces in younger and older adults. Neuropsychologia, 129, 10-20. 